\title{
Effect of dehydroepiandrosterone (DHEA) on monoamine oxidase activity, lipid peroxidation and lipofuscin accumulation in aging rat brain regions
}

\author{
Pardeep Kumar · Asia Taha · Deepak Sharma • \\ R. K. Kale $\cdot$ Najma Z. Baquer
}

Published online: 3 June 2008

(C) Springer Science+Business Media B.V. 2008

\section{Erratum to: Biogerontology \\ DOI 10.1007/s10522-008-9133-y}

This erratum is published as discrepancies related with table captions were noticed in the original publication (Tables 2 and 3).

The online version of the original article can be found under doi:10.1007/s10522-008-9133-y.

P. Kumar · A. Taha · D. Sharma · N. Z. Baquer $(\square)$

Neurobiology Laboratory School of Life Sciences, Jawaharlal Nehru University, New Delhi 110067, India e-mail: nzbaquer@hotmail.com; nzbaquer@gmail.com

P. Kumar · R. K. Kale

Cancer and Radiation Biology Laboratory, School of Life

Sciences, Jawaharlal Nehru University,

New Delhi 110067, India 
Table 2 Monoamine oxidase activity in synaptosomal and supernatant fractions in cerebral hemispheres of 4, 14 and 24 months of control and DHEA treated aging male rats

\begin{tabular}{|c|c|c|c|c|}
\hline \multicolumn{5}{|c|}{ Monoamine oxidase activity (U/mg protein/min) } \\
\hline \multirow{2}{*}{$\begin{array}{l}\text { Age in } \\
\text { months }\end{array}$} & \multicolumn{2}{|c|}{ Synaptosomal fraction } & \multicolumn{2}{|c|}{ Supernatant fraction } \\
\hline & Control & DHEA & Control & DHEA \\
\hline 4 & $1.25 \pm 0.02$ & $1.24 \pm 0.02$ & $0.259 \pm 0.02$ & $0.257 \pm 0.02$ \\
\hline 14 & $3.28 \pm 0.03$ & $2.71 \pm 0.02^{\mathrm{b}}$ & $1.16 \pm 0.03$ & $1.02 \pm 0.02^{\mathrm{c}}$ \\
\hline 24 & $3.84 \pm 0.08^{\alpha}$ & $2.16 \pm 0.20^{\mathrm{a} \beta}$ & $1.37 \pm 0.008^{\alpha *}$ & $0.71 \pm 0.03^{\mathrm{a} \beta}$ \\
\hline
\end{tabular}

Each value is a mean of \pm SEM of five separate experiments. Statisitical analysis done by two-way ANOVA followed by bonferroni test. $p$-values are: ${ }^{\mathrm{a}} p<0.001,{ }^{\mathrm{b}} p<0.01,{ }^{\mathrm{c}} p<0.05$. Comparing age matched controls vs. DHEA treatments $(p<0.001)$ comparing in controls and in DHEA treated animals with aging when compared with young (4 months)

$\left({ }^{\alpha} p<0.001\right)\left({ }^{*} p<0.05\right)$ Significant differences among controls age groups. $\left({ }^{\beta} p<0.001\right)$ Significant differences among in DHEA treated age groups

Table 3 Malondialdehyde (MDA) levels in whole homogenates of 4, 14 and 24 months of control and DHEA treated aging male rat brain regions

\begin{tabular}{|c|c|c|c|c|c|c|}
\hline \multirow{4}{*}{$\begin{array}{l}\text { Age in } \\
\text { months }\end{array}$} & \multicolumn{6}{|l|}{ Brain regions } \\
\hline & \multicolumn{2}{|c|}{ Cerebral hemisphere } & \multicolumn{2}{|l|}{ Cerebellum } & \multicolumn{2}{|l|}{ Brain stem } \\
\hline & \multicolumn{6}{|c|}{ nmoles of $\mathrm{MDA} / \mathrm{mg}$ protein } \\
\hline & Control & DHEA & Control & DHEA & Control & DHEA \\
\hline 4 & $0.317 \pm 0.034$ & $0.266 \pm 0.033$ & $0.284 \pm 0.015$ & $0.274 \pm 0.026$ & $0.268 \pm 0.012$ & $0.242 \pm 0.012$ \\
\hline 14 & $0.927 \pm 0.04$ & $0.604 \pm 0.02^{\mathrm{b}}$ & $0.919 \pm 0.029$ & $0.785 \pm 0.017^{\mathrm{b}}$ & $0.763 \pm 0.012$ & $0.606 \pm 0.010^{\mathrm{c}}$ \\
\hline 24 & $1.29 \pm 0.061^{\alpha}$ & $0.598 \pm 0.015^{\mathrm{a} \beta}$ & $1.15 \pm 0.057^{\alpha \$}$ & $0.554 \pm 0.053^{\mathrm{a} \beta}$ & $0.977 \pm 0.082^{\alpha *}$ & $0.698 \pm 0.011^{\mathrm{a} \beta}$ \\
\hline
\end{tabular}

Each value is a mean of \pm SEM of five separate experiments. Statisitical analysis done by two-way ANOVA followed by bonferroni test. $p$-values are: ${ }^{\mathrm{a}} p<0.001,{ }^{\mathrm{b}} p<0.01,{ }^{\mathrm{c}} p<0.05$. Comparing age matched controls vs. DHEA treatments $(p<0.001)$ comparing in controls and in DHEA treated animals with aging when compared with young (4 months)

$\left({ }^{\alpha} p<0.001\right)\left({ }^{\$} p<0.01\right)\left({ }^{*} p<0.05\right)$ Significant differences among controls age groups. $\left({ }^{\beta} p<0.001\right)$ Significant differences among in DHEA treated age groups 